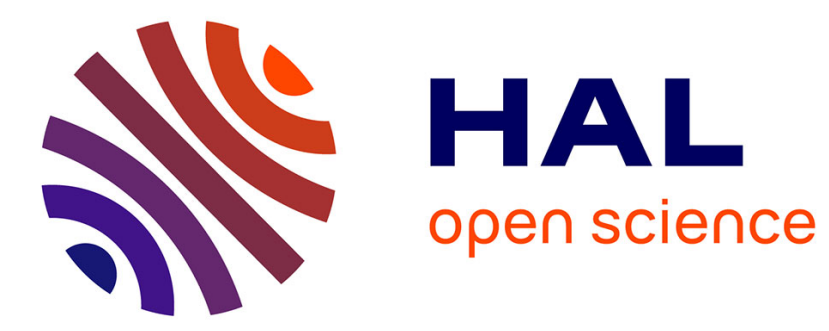

\title{
EFFETS NON LINÉAIRES DE LA RELAXATION DE BORDONI ET ÉVOLUTION DES CONTRAINTES INTERNES
}

\author{
C. Esnouf, J. Chicois, A. Hamel, R. Fougères, Gilbert Fantozzi
}

\section{To cite this version:}

C. Esnouf, J. Chicois, A. Hamel, R. Fougères, Gilbert Fantozzi. EFFETS NON LINÉAIRES DE LA RELAXATION DE BORDONI ET ÉVOLUTION DES CONTRAINTES INTERNES. Journal de Physique Colloques, 1983, 44 (C9), pp.C9-665-C9-671. 10.1051/jphyscol:19839100 . jpa-00223333

HAL Id: jpa-00223333

https://hal.science/jpa-00223333

Submitted on 1 Jan 1983

HAL is a multi-disciplinary open access archive for the deposit and dissemination of scientific research documents, whether they are published or not. The documents may come from teaching and research institutions in France or abroad, or from public or private research centers.
L'archive ouverte pluridisciplinaire $\mathbf{H A L}$, est destinée au dépôt et à la diffusion de documents scientifiques de niveau recherche, publiés ou non, émanant des établissements d'enseignement et de recherche français ou étrangers, des laboratoires publics ou privés. 


\title{
EFFETS NON LINEAIRES DE LA RELAXATION DE BORDONI ET ÉVOLUTION DES CONTRAINTES INTERNES
}

\author{
C. Esnouf, J. Chicois, A. Hame1, R. Fougères et G. Fantozzi \\ Groupe d'Etudes de Métallurgie Physique et de Physique des Matériaux*, \\ I.N.S.A., 69621 Vizzeurbanne Cedex, France
}

\begin{abstract}
Résumé - Nous avons êtudié, dans le cas d'un aluminium 5N, I'évolution de $1 a$ relaxation non linéaire de Bordoni en fonction de 1a température de recuit après déformation plastique. De ces résultats et de l'effet d'une contrainte statique sur le frottement intérieur, on déduit l'évolution des contraintes internes au cours du recuit. Cette évolution avec la température est similaire à celle observée sur des valeurs de contrainte interne obtenues par la méthode du dip-test, bien que les niveaux de contrainte observés soient différents.

Abstract - Evolution of non linear Bordoni relaxation has been studied as a function of annealing temperature of $5 \mathrm{~N}$ aluminium plastically deformed. From these results and from the effect of applied stress on internal friction the evolution of internal stresses with annealing is deduced. This evolution is similar to this obtained by dip-test measurement although the stress levels are different.
\end{abstract}

\section{I - INTRODUCTION}

Dans une précédente étude $/ 1 /$, nous avions mis en évidence une corrêlation entre les propriétés non linéaires de la relaxation de Bordoni et le niveau de contraintes internes mesurées directement par la méthode du dip-test. En fonction de 1 'amplitude de la déformation plastique préalable, nous avions observé un effet de la contrainte appliquée sur la hauteur du pic de Bordoni d'autant plus faible que le niveau de contrainte interne était moins êlevé. Cependant, les niveaux des contraintes internes mesurés après déformation plastique et ceux de la contrainte apoliquée permettant de remplir la condition de Paré, étaient diffërents d'environ un ordre de grandeur. La présente étude cherche à établir une corrélation entre contrainte interne et prooriétés non linéaires de la relaxation de Bordoni dans le cas d'une restauration des contraintes internes par recuits successifs d'états préalablement déformés. L'exoérimentation effectuée sur un même échantillon autorise à déduire đes variations de frottement intérieur 1 'évolution des contraintes internes avec la température de recuit calculée à partir d'un modèle simple à deux puits. I1 est alors oossible d'établir une corrélation entre les niveaux de contrainte interne mesurés et calculẻs.

\section{II - RESULTATS EXPERIMENTAUX}

Le matêriau utilisé est 1 'aluminium de pureté $5 \mathrm{~N}$, les échantillons étant communs aux deux types d'essai : le dip-test et le frottement intérieur. Les mesures du décrément logarithmique ont été effectuêes sur un pendule inversé automatique utilisant une frëquence de vibration proche de $1^{\text {'hertz. }}$

Tous les échantillons sont dêformés en torsion bidirectionne11e de 0,5\% effectuée deux fois à $10 \mathrm{~K}$ ou à $300 \mathrm{~K}$ et subissent des recuits à températures variables.

\footnotetext{
*Laboratoire associé au C.N.R.S. n 341
} 
Pour chaque état, on procède à l'enregistrement des pics de Bordoni (T. $\epsilon 10-250 \mathrm{~K})$ avec une amplitude de sollicitation $\varepsilon=4,5 \cdot 10^{-6}$, puis $1^{\prime}$ opération est répétée en superposant à $\varepsilon$ une contrainte statique $\sigma_{S}$ de $3 \cdot 10^{-5} \mu$ ( $\mu$ : module de cisaillement). Jn exemple d'enregistrement est donné par la figure 2 : on reconnaît le pic de Niblett et Willes $B_{1}$, situé vers $70 \mathrm{~K}$, et le pic de Bordoni $B_{2}$ autour de $100 \mathrm{~K}$. Un accroissement impoitant de l'intensité de relaxation des deux pics est observé en présence de la contrainte $\sigma_{S}$. Afin de préciser exactement cet effet non linéaire, nous procédons à l'êlimination du fond de frottement intērieur de la manière suivante : côté basse, puis haute températures de la relaxation de Bordoni, un fond $\delta_{f}$ est soustrait en choisissant une relation linéaire entre $\log \delta_{\mathrm{F}}$ et $1 / \mathrm{T}$. Le rapport $\mathrm{R}$ entre les hauteurs du pic $B_{2}$ avec et sans la contrainte $\sigma_{S}$ est alors calculé. Son évolution avec

les traitements chọisis est prêsentée par 1a figure 3. Pour les deux types de traitements, le rapport $R$ croît avec la température de recuit, excepté pour la température de $415 \mathrm{~K}$. Dans ce cas, les impuretés ont migré vers les dislocations, diminuant considérablement 1 a hauteur du pic de Bordoni et rendant la mesure du rapport $R$ assez imprécise. Globalement, on peut dire que le caractère non linéaire de la relaxation augmente avec la température de recuit.

\section{III - ANALYSE DES RESULTATS}

Comme cela a été discuté longuement dans là littérature $/ 2,3,4 /$, 1e caractère non $1 i-$ néaire de la relaxation de Bordoni est essentiellement attribué au non respect de la condition de Paré. Si on considère le diagramme énergétique d'une 1igne de dislocation dont le mouvement est thermiquement activé (Fig. 4a). La condition de Paré est respectée lorsqu'au moins les énergies correspondant aux deux premières positions sont égales. Cette condition peut être obtenue à I'aide des contraintes internes ou d'une contrainte statique. On notera $\sigma_{0}$ la contrainte de Paré permettant le respect de cette condition.

Deux situations sont alors rencontrées : celle figurée par la courbe 1 de la figure $4 a$ où les contraintes sont inférieures à $\sigma_{0}$ et celle figurée par la courbe 2 où elles sont supérieures à $\sigma_{0}$. La deuxième situation mène à un comportement linéaire de la relaxation vis à vis de la contrainte. L'intensité de relaxation est alors maximale et de $l^{\prime}$ ordre de $\Lambda \ell^{2} / 10$ ( $\Lambda$ et $\ell$ sont respectivement la densité et la longueur des đislocations). La première situation peut être modélisée en ne gardant que les deux premiers puits (Fig. 4b). En effet, la majorité des dislocations occupe les puj.ts 0 ou 1, 1a probabilité d'occupation décroissant exponentiellement /3/ avec 1 'écart d'énergie $\delta \mathrm{E}=\mathrm{E}_{\mathrm{D}}-\mathrm{E}_{\mathrm{G}}$ (ici $\delta \mathrm{E}>0$ pour toutes les vallées). $\mathrm{E}_{\mathrm{D}}$ est $I^{\prime}$ énergie de $1 \mathrm{a}$ barrière dans le sens 0 vers $l$ et $E_{G}$ pour le sens inverse. Ce modèle simple permet le calcul de l'intensité de relaxation en fonction de l'écart ó en utilisant la théorie cinétique :

$$
\frac{\mathrm{dn}_{0}}{\mathrm{dt}}=-\frac{\mathrm{dn}}{\mathrm{dt}}=\mathrm{k}_{10} \mathrm{n}_{1}-\mathrm{k}_{01} \mathrm{n}_{0} \quad \text { avec } \quad \mathrm{n}_{0}+\mathrm{n}_{1}=1
$$

$k_{0 I}$ et $k_{10}$ sont les probabilitês de saut de 0 vers 1 ou 1 vers 0 , $n_{0}$ et $n_{1}$ les probabilités d'occupation $\left(k_{01}=k_{0} \exp \left(-\frac{E_{D}}{k T}\right) ; k_{10}=k_{0} \exp \left(-\frac{E_{T}}{k T}\right) ; k\right.$ (Eréquence d'attaque de la dislocation.). A I'équilibre, c'est-à-dire en présence des seules contraintes internes $\sigma_{i}, \frac{\mathrm{dn}_{0}}{\mathrm{~d} t}=0$ et ainsi $\mathrm{n}_{0}=\mathrm{n}_{0}^{e}=\frac{\mathrm{k}_{10}}{\mathrm{k}_{01}+\mathrm{k}_{10}} ; \mathrm{n}_{1}=\mathrm{n}_{1}^{e}=\frac{\mathrm{k}_{01}}{\mathrm{k}_{01}+\mathrm{k}_{10}}$.

En présence de la contrainte de mesure $\sigma$, les probabilités de saut sont modifiées : il suffit de remplacer $\mathrm{E}_{\mathrm{D}}\left(\sigma_{i}\right)$ par $\mathrm{E}_{\mathrm{D}}\left(\sigma_{i}+\sigma\right)$ (de même pour $\mathrm{E}_{\mathrm{G}}$ ), soit :

$\mathrm{E}_{\mathrm{D}}\left(\sigma_{\mathrm{i}}\right)+\frac{\partial \mathrm{E}_{\mathrm{D}}}{\partial \sigma} \cdot \sigma$, dans 1 'hypothèse d'une variation linéaire de $\mathrm{E}_{\mathrm{D}}$, avec $\sigma$ (ou volume d'activation constant) sur le domaine $\left[-\sigma_{m},+\sigma_{m}\right]\left(\sigma_{m}\right.$ : valeur maximale de $\left.\sigma\right)$. 
Si on pose $\frac{\partial E_{D}}{\partial \sigma}=-v_{D}$ et $\frac{\partial E_{G}}{\partial \sigma}=+v_{G}$, les nouvelles probabilités de saut sont :

$$
\begin{gathered}
k_{01}^{\prime}=k_{0} \exp -\left(\frac{E_{D}\left(\sigma_{i}\right)-v_{D} \sigma}{k T}\right) \approx k_{0} \exp -\left(\frac{E_{D}\left(\sigma_{i}\right)}{k T}\right)\left(1+\frac{v_{D} \sigma}{k T}\right), \\
k_{01}^{\prime} \approx k_{01}\left(1+\frac{v_{D} \sigma}{k T}\right) \quad \text { et } \quad k_{10} \simeq k_{01}\left(1-\frac{v_{G} \sigma}{k T}\right) .
\end{gathered}
$$

L'évolution des grandeurs $n_{0}$ et $n_{l}$ permet de connâtre 1'aire balayêe A par les dislocations et donc la déformation $\varepsilon_{\mathrm{d}}$ associée :

$$
E_{d}(t)=N A(t) b=\Lambda a b\left(n_{1}(t)-n_{1}^{e}\right) \quad(a: \text { distance entre les puits })
$$

Le système (1) et 1 'équation (2) permettent d'écrire :

$$
\dot{\varepsilon}_{\mathrm{d}}=\Lambda \mathrm{ab} \frac{\mathrm{v}_{\mathrm{D}}+\mathrm{v}_{\mathrm{G}}}{\tau^{\prime}} \cdot \frac{\sigma}{\mathrm{kT}}-\frac{\varepsilon_{\mathrm{d}}}{\tau} \text {, }
$$

où

$$
\frac{1}{\tau}=k_{01}^{\prime}+k_{10} \simeq k_{01}+k_{10} \quad \text { et } \quad \frac{1}{\tau^{\prime}}=\frac{k_{01} k_{10}}{k_{01}+k_{10}} \text {. }
$$

L'expression du déphasage entre la déformation totale $\varepsilon(t)=\frac{\sigma}{\mu}+\varepsilon_{\mathrm{d}}$ et la contrainte donne le frottement intérieur sous la forme :

$$
\delta=\pi \frac{\tau}{\tau} \Lambda \text { \&a } \frac{\mu \mathrm{b}^{3}}{\mathrm{kT}} \cdot \frac{\omega \tau}{1+\omega^{2} \tau^{2}} \quad(\omega: \text { pulsation }) .
$$

L'intensité de relaxation est alors proportionnelle au rapport $\tau / \tau^{\prime}$, c'est-à-dire :

$$
\delta \alpha n_{0} n_{1}^{e}=\frac{1}{2\left(1+C h \frac{\sigma E}{k T}\right)} .
$$

Cependant, les expressions de $E_{D} \simeq 2 W_{k}$ (énergie de création des doubles décrochements à 1 'origine du pic de Bordoni) et de $\mathrm{E}_{\mathrm{G}} \simeq \sigma_{i}$ abl (travail de 1 a contrainte interne)
montrent que :

$$
\delta E \simeq 2 W_{k}-\sigma_{i} a b l
$$

évolue avec $\sigma_{i}$, mais aussi la longueur des dislocations.

Maintenant, la superposition aux contraintes $\sigma_{i} d$ 'une contrainte statique $\sigma_{S}$ fait que le rapport des intensitês de relaxation s'écrit :

$$
\frac{{ }_{\sigma_{S}}}{\delta_{\sigma_{S}=0}}=\frac{1+\operatorname{Ch}(\delta E / k T)}{1+C h\left(\left(\delta E-\sigma_{S} a b 2\right) / k T\right)} .
$$

Cette expression devrait décrire les phênomènes non linéaires observês, mais elle n'est valable qu'avec $\delta E>0$, c'est-à-dire si $\sigma_{i}<\sigma_{0}$. Pour éviter cette limitation, i1 convient de tenir compte de la distribution des longueurs de dislocations donnée par la probabilité $n(l) d \ell=\frac{\ell}{\ell^{2}} \exp \left(-\ell / \ell_{0}\right) \mathrm{d} l$, où $\ell_{0}$ est la longueur moyenne de la distribution /5/. En posant : $\sigma_{0} a b \ell_{0}=2 \mathrm{~W}_{\mathrm{k}}, 1^{\prime}$ expression (7) devient :

$$
\delta E=2 W_{k}\left(1-\frac{\sigma_{i} \ell}{\sigma_{0} \ell_{0}}\right),
$$


avec $\omega \tau_{0} \exp \left(\frac{2 W k}{k T_{M}}\right)=1$ si $T_{M}$ est la température du pic de Bordoni et $\tau_{0}$ le terme proportionne $\simeq 10^{-11} \mathrm{~s} / 6 /$. Ainsi :

$$
\frac{\delta \mathrm{E}}{\mathrm{kT}_{\mathrm{M}}} \simeq-23\left(1-\frac{\sigma_{i}}{\sigma_{0}} \frac{\ell}{\ell_{0}}\right),
$$

dans le domaine de 1 'hertz et l'intensité de relaxation, est alors proportionnelle à

$$
F\left(\sigma_{i} / \sigma_{0}\right)=\frac{1}{2} \int f(x) \cdot x e^{-x} d x
$$

sur le domaine $\mathrm{x}=\frac{\ell}{\ell_{0}} \in\left[0, \mathrm{x}_{\mathrm{m}}=\left(\sigma_{0} / \sigma_{i}\right)\right]$, avec :

$$
\begin{array}{ll}
f(x)=\frac{1}{1+C h\left(23\left(1-\frac{\sigma_{i}}{\sigma_{0}} x\right)\right)} & x<x_{m} \\
f(x)=\frac{1}{4} & x>x_{m}
\end{array}
$$

Le rapport des intensités de relaxation avec et sans contrainte statique est ainsi donné par :

$$
\mathcal{R}=\mathrm{F}\left(\frac{\sigma_{i}+\sigma_{s}}{\sigma_{0}}\right) / F\left(\frac{\sigma_{i}}{\sigma_{0}}\right) \text {. }
$$

I1 est essentiellement fonction des contraintes interne et statique relatives à la contrainte de Paré $\sigma_{0}$, elle-même déterminée par la longueur moyenne $\iota_{0}$. La figure 5 présente 1 'évolution de cette fonction $\mathrm{F}^{\prime}$.

$\mathrm{I} 1$ convient à présent de $I^{\prime}$ utiliser en cherchant la valeur $\sigma_{i}$ qui donne $\mathbb{R}=\mathrm{R}$ (rapport expérimental). Cette recherche est effectuée pour différentes valeurs de $\sigma_{0}$ (ou $\left.\ell_{0}\right)$ lorsque $\bar{l}$ e produit $\ell_{0} \sigma_{0}=2 \mathrm{~W}_{\mathrm{k}} / \mathrm{ab} \simeq \frac{\mu b}{207}\left(2 \mathrm{~W}_{\mathrm{k}} \simeq 0,2 \mathrm{eV}\right)$ et lorsque $\sigma_{\mathrm{S}}=3 \cdot 10^{-5} \mu$. Un
exemple est présenté sur la figure 5.

L'ensemble des résultats est exposé sur la figure 6 pour quelques valeurs de $\ell_{0}$.

IV - DISCUSSION

L'examen de la figure 6 montre que les contraintes internes diminuent avec la température de recuit, indiquant une réorganisation du réseau de dislocations dès les premiers recuits. On constate également que les contraintes internes sont un peu plus faibles après une déformation à I'ambiante par rapport à une déformation à $10 \mathrm{~K}$, suivie d'un recuit à l'ambiante.

La comparaison de ces résultats avec ceux obtenus par la méthode du dip-test indique qu'ils sont globalement semblables. Cependant, quelques remarques peuvent être faites : le niveau de contraintes internes mesuré par frottement intêrieur est plus faible à moins d'admettre $l$ 'existence de très courtes dislocations ( $\ell_{0}$ de $l$ 'ordre de quelques b). Compte tenu de 1 'importante intensité de relaxation mesurée $\left(\delta \simeq 3.10^{-2}\right)$, il semble que cela ne puisse être le cas. Les deux méthodes ne mettent donc pas en évidence les mêmes contraintes.

D'autre part, la valeur obtenue après recuit à $415 \mathrm{~K}$ est ici peu significative ; la longueur des dislocations y est très faible, le rapport $\sigma_{\mathbf{S}} / \sigma_{0}$ également. La solution nécessite une connaissance très exacte de la fonction $F$, d'autant plus que l'écart $\sigma_{S} / \sigma_{0}$ est faible. L'examen de la fonction $f(x)$ (rel. 10) montre que la solution est surtout déterminée par les grandes valeurs de $x$, c'est-à-dire les grandes longueurs de 1a distribution et donc sensible à la forme de la distribution.

Enfin, il y aurait lieu de tenir compte d'une variation de la longueur moyenne $\ell_{0}$ dès que $\sigma_{i}$ évolue, mais le problème théorique est trop ardu. 


\section{$V$ - CONCLUSION}

Comme après écrouissage, on observe, dans le cas de recuits successifs d'état préalablement déformés, une évolution similaire des contraintes internes mesurées par la méthode du dip-test et celles déduites des propriétés non linéaires de la relaxation de Bordoni. Cependant, les niveaux mesurés sont ici encore très supérieurs à ceux que l'on peut déduire par calcul à partir d'hypothèses vraisemblables sur la longueur libre moyenne des dislocations. Cette différence de valeur accrédite 1 'idée selon laquelle les contraintes internes sont distribuées. En effet, la relaxation de Bordoni est due principalement aux dislocations de grandes longueurs et donc soumises aux contraintes internes les plus faibles alors que la méthode du dip-test donne une valeur moyenne de la distribution. Cependant, il faut noter qu'elles évoluent d'une manière similaire entre elles, aussi bien après écrouissage qu'après recuit.

\section{REFERENCES}

/1/ CHICOIS J., HAMEL A., FOUGERES R., ESNOUF C., FANTOZZI G. and PEREZ J., Journal de Physique, colloque C5, supplémentaire $\mathrm{n}^{\circ}$ 10, tome 42, octobre 1981 .

/2/ PARE V.K., Thèse Corne11 University, Ithaca, N.Y., 1958.

/3/ ESNOUF C., Thèse, Université Lyon 1, I.N.S.A., 1978.

14/ ESNOUF C. and FANTOZzI G., Phys. Stat. (a) 47, 201, 1978.

/5/ KOEHLER J.S., Imperfections in nearly perfect crystals, J. Wiley, 197, 1969.

/6/ FANTOZZI G., ESNOUF C., BENOIT W. and REICHIE J.g., Bordoni relaxation, Progress in Mat. Sci., 27, 311, 1982 . 


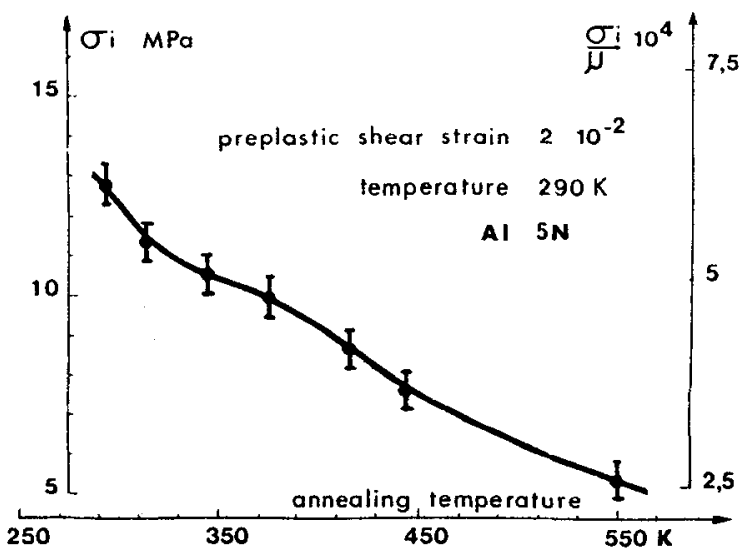

Fig. 1 : Contraintes internes mesurées par la méthode du dip-test en fonction des recuits (1 heure).

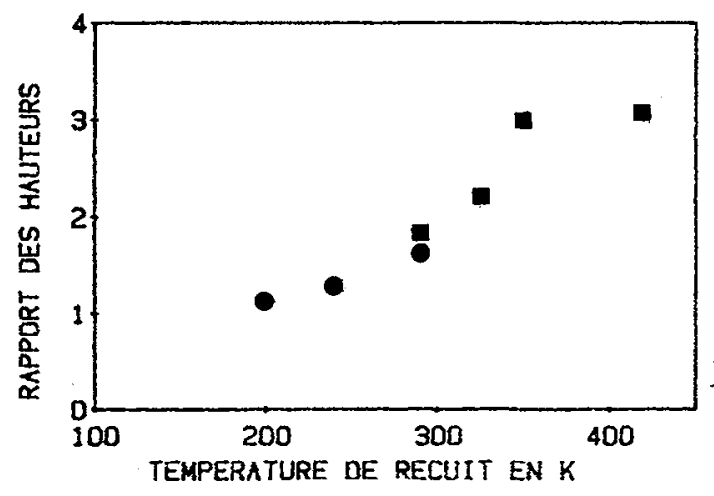

Fig. 3 : Evolution du rapport $\mathrm{R}$ au cours des recuits.

(-) déformation à $210 \mathrm{~K}$

a: déformation à $290 \mathrm{~K}$ ).
Fig. 4 : Diagramme ênergiedéplacement d'une dislocation en présence des barrières de Peïerls (a) : importance de la contrainte interne

(b) : modèle à deux puits.

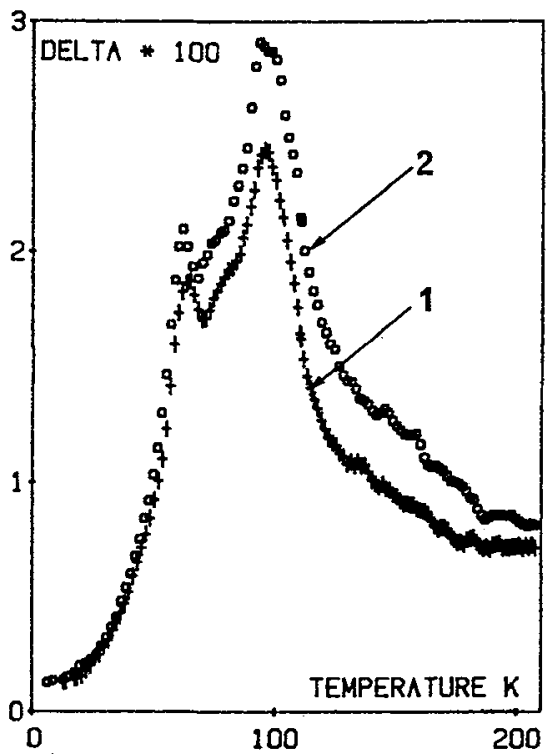

Fig. 2 : Spectres de frottement intérieur sans (1) et avec (2) Ia contrainte statique $\sigma_{\mathrm{S}}=3 \cdot 10^{-5} \mathrm{\mu}$ (déformation à $10 \mathrm{~K}$ et recuit à $\left.240 \mathrm{~K}, \varepsilon=4,5 \cdot 10^{-6}\right)$.

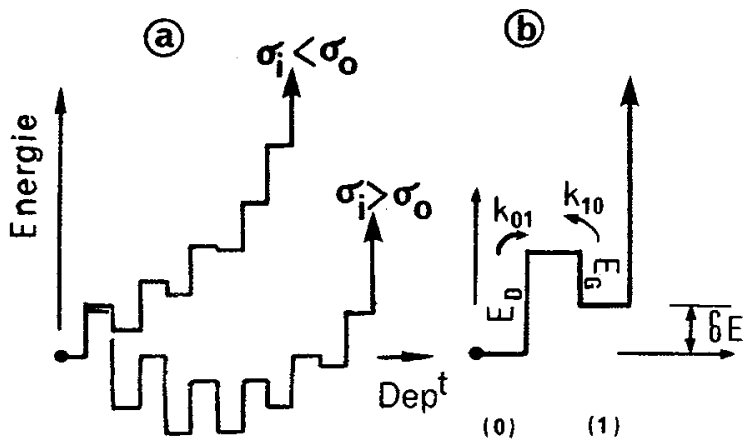



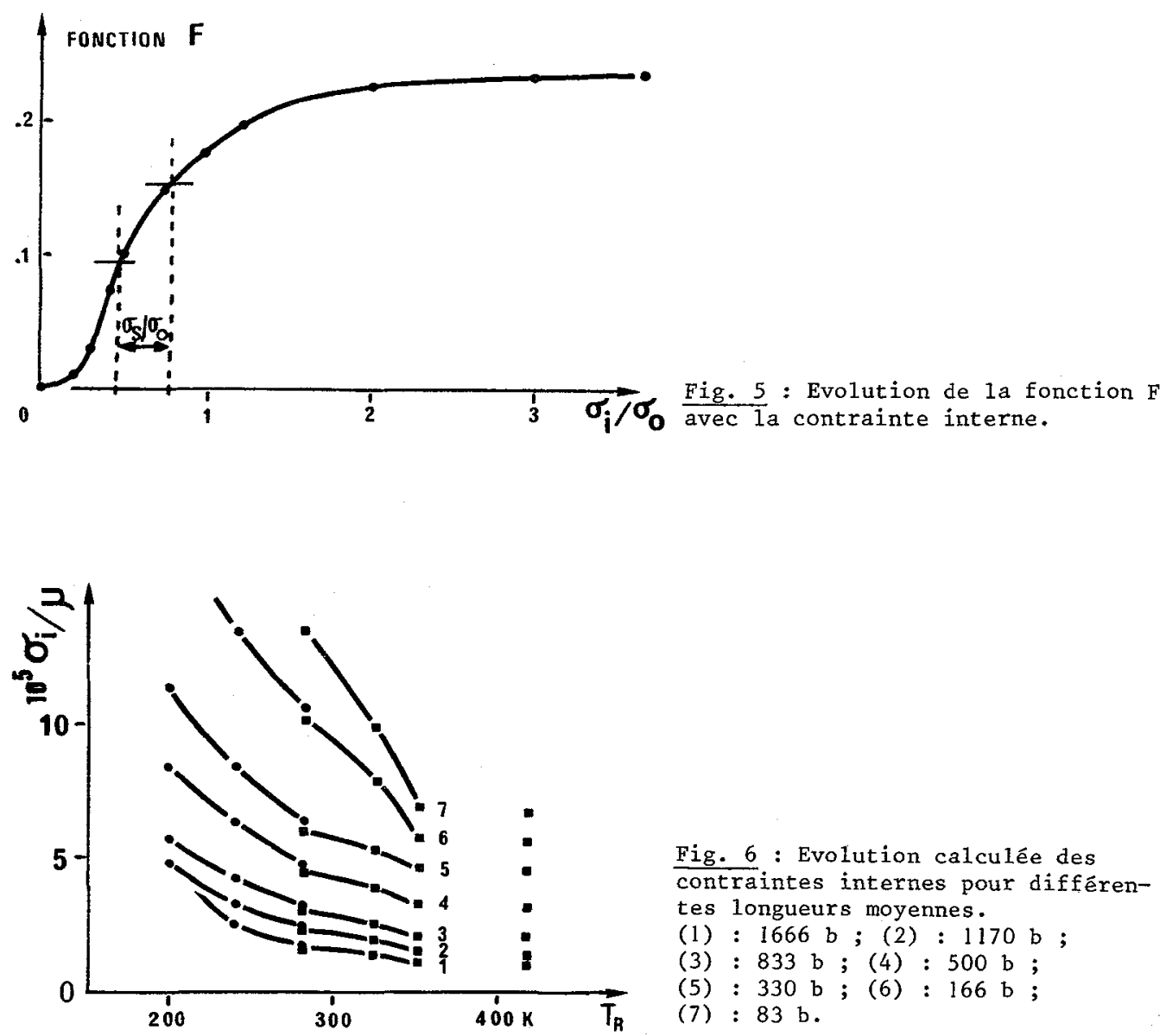

Fig. 6 : Evolution calculée des contraintes internes pour différentes longueurs moyennes.

(1) : $1666 \mathrm{~b} ;(2): 1170 \mathrm{~b}$;

(3) : $833 \mathrm{~b} ;(4): 500 \mathrm{~b}$;

(5) : $330 \mathrm{~b} ;(6): 166 \mathrm{~b}$;

(7) : $83 \mathrm{~b}$. 